\title{
Severe hypernatremic dehydration leading to bilateral lower limb ischemia in a neonate
}

Liyanage $\mathrm{PN}^{1}$, Hettiarachchi $\mathrm{RM}^{1}$

\section{Introduction}

Peripheral limb ischaemia resulting in gangrene is rare in children. We present a case of thromboembolism leading to limb ischemia secondary to hypernatremic dehydration requiring life saving bilateral lower limb amputation in a 12day-old neonate.

\section{Case report}

A 12 day-old baby boy was brought to Neonatal Intensive Care Unit of General Hospital Matara with bilateral bluish discoloration of lower limbs below the ankles. Over next 12 hours bluish discolorations progressed to involve up to the knee level.

The baby was born at term with a birth weight of $3.3 \mathrm{~kg}$ by an elective Caesarian section to a 34 years old Rhesus A negative mother who had type 2 diabetes mellitus. Intramuscular $1 \mathrm{mg}$ of vitamin $\mathrm{K}$ was given soon after delivery. Rhesus immune globulin was administered to mother before discharge. Despite advice from field family health worker baby was feeding poorly at home. Baby remained weak and lethargic. On day 12 parents decided to seek medical attention.

On examination his weight was $2.4 \mathrm{~kg}$ (Birth weight 3.3. kg), drowsy and floppy with a poor cry. He was afebrile and not pale. Heart rate was 170 beats per minute (normal for the age) with a soft systolic cardiac murmur. Dorsalis pedis and posterior tibial pulses were not palpable in both legs. Femoral pulses were weakly felt. Saturation of oxygen was not recordable in both feet. Respiratory rate was 52 breaths per minute. Abdominal examination was normal apart from a mildly enlarged liver. Temperature remains normal. Gangrenous limbs were noted.

Initial resuscitation included intravenous fluids, oxygen, inotropes and parenteral antibiotics.

ECG axis was deviated to right and tall $T$ waves were detected. Echocardiogram revealed a small muscular ventricular septal defect and an ostium secondum atrial septal defect. Right atrial and ventricular dilatation with mild global hypokinasea was noted. There were no intra cardiac clots. White cell count was $19100 / \mathrm{mm}^{3}$ with $52 \%$ lymphocytes $C$ reactive protein was undetected and blood cultures remained sterile. Platelet count was

1Neonatology Unit District General Hospital Matara
$70,000 / \mathrm{mm}^{3}$. INR was 2.46 and APTT was $46 \mathrm{sec}$. Serum sodium was $187 \mathrm{meq} / \mathrm{L}$ and serum potassium was 8.8 $\mathrm{meq} / \mathrm{L}$. Serum creatinine was $6.6 \mathrm{mg} / \mathrm{dL}$.

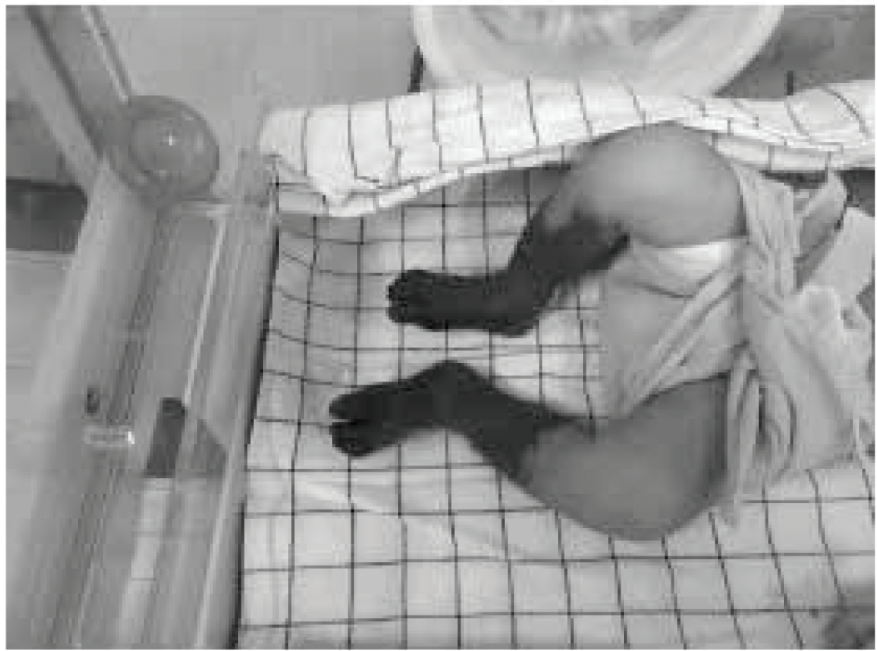

Considering nearly a $25 \%$ of weight loss and elevated serum sodium, urea and creatinine values a clinical diagnosis of hypernatraemic dehydration was made. Doppler scan revealed thrombosis in the aorta below the renal artery level extending to the bifurcation and there was no detectable blood flow in both femoral arteries. Cranial ultra sound excluded venous sinus thrombosis.

Intravenous (IV) heparin infusion was started. Baby was transferred to Teaching Hospital Karapitiya. Gangrenous limbs required life saving below knee amputation and baby was discharged with warfarin therapy after 40 days of hospital stay.

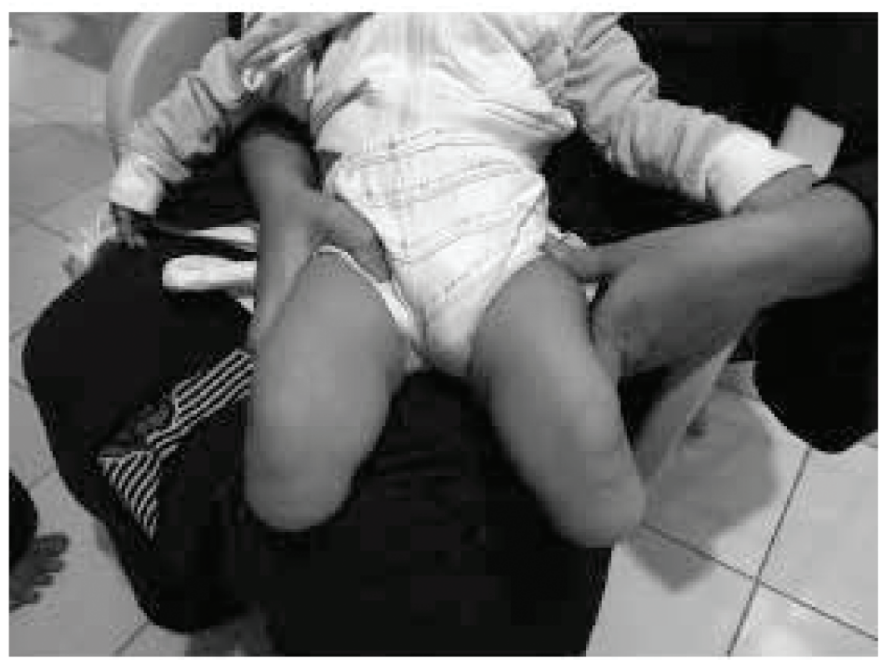




\section{Discussion}

Gangrene of the extremities is extremely rare at birth. Neonatal limb ischemia may have considerable challenges in diagnosis and management. The $1^{\text {st }}$ case report on bilateral symmetrical lower limb gangrene was from Southern Africa ${ }^{1}$.

Causes for the limb ischemia in neonates are many. Sepsis and hyper coagulable states head the list. Polycythemia, hypernatremia, hyperglycemia, maternal diabetes, extravasations of IV fluid, IV hyperosmolar infusion all have been reported as causes for neonatal limb ischemia 2 .

The pathophysiology of these events, in the context of the neonatal haemostatic system remains poorly defined. In our case, dehydration was responsible of thrombosis, and it was also secondary to maternal lactation failure, poor fluid intake and increased insensible water loss. Severe neonatal hypernatremia is an important electrolyte disorder that has serious effects. Cerebral venous thrombosis and aortic thrombosis are relatively rare in severe neonatal hypernatremic dehydration ${ }^{3}$.

Other conditions such as congenital rubella, varicella and necrotizing fasciitis which may present in a similar fashion should be considered in differential diagnosis.

Severe symptomatic thromboembolic events are typically treated with anticoagulants and/or fibrinolytic agents. Surgical thrombectomy is rarely performed in newborn. If medical management fails to improve the perfusion to the leg, then surgical thrombectomy or embolectomy should be considered ${ }^{4}$. In case of established gangrene, amputation is the treatment of choice ${ }^{5}$. The aim should be to optimize future reconstruction and rehabilitation. It is important for the orthopaedic surgeon to be involved in the treatment and planning as soon as gangrene is observed in children
Authors acknowledge the support and expertise of clinicians of all the teams at DGH Matara and TH Karapitiya who were involved in management of this patient.

\section{References}

1. Michael Gelfand, Government Medical Officer, Salisbury Native Hospital, Southern Rhodesia, Southern Africa, Symmetrical Gangrene in the Africans, 1947 Jun 14; 1(4510):847-849.

2. Seth T. Thrombosis in neonates and children. E J Med 2009;14:36-45

3. Hbibi M, Abourazzak S, Babakhouya A, et al. Severe hypernatremic dehydration associated with cerebral venous and aortic thrombosis in the neonatal period BMJ Case Rep. 2012; 2012: bcr0720114426.

4. Quddsi Al, Nizami N., Razzaq A., Hussain S., Bilateral gangrene of lower limbs in a neonate, Journal of the College of Physicians \& Surgeons, Pakistan 2014, Vol 24 (September)

5. Letts M, Blastorah B, al-Azzam S, Neonatal Gangrene of the Extremity: A Report of Three Cass and Review of the Literature. Journal of Pediatric Surgical Specialties, 17(03)(1997), pp.397-401 